\title{
ENSEÑANZA DE LA DIFERENCIA ÉTNICO-RACIAL COLOMBIANA EN LAS CIENCIAS SOCIALES: UNA MIRADA A LOS APORTES DEL CAMPO DE ESTUDIO DE LA COMUNICACIÓN-EDUCACIÓN
}

(Teaching Colombian ethno-racial difference in the social sciences: a look at the contributions of the field of study of communicationeducation)

Adriana Hernández Bocanegra' adrhernandezb@hotmail.com

Fecha de recepción: 20 febrero de $2015 . \quad$ Fecha de aceptación: 7 abril de 2015.

Reflexionar sobre los modos de asumir la diversidad étnico-racial en instituciones educativas, implica abrir un espacio al debate sobre cómo se comprende la diferencia cultural desde la impronta étnico-racial en términos teóricos y en sus manifestaciones cotidianas. Al respecto, se hacen evidentes dos importantes tendencias: la primera parte del precepto de la cultura como una categoría homogénea históricamente definida; la segunda, reconoce el carácter cambiante de la cultura y de los sujetos que forman parte de ella. En ese sentido, el presente documento explora las tendencias que orientan el debate sobre la enseñanza de la diferencia étnico-racial colombiana, haciendo énfasis en los sujetos, prácticas escolares y saberes, que inciden principalmente en la formación de representaciones sociales. Asimismo, se plantea la importancia de la comunicación-educación como eje articulador de los procesos de diálogo intercultural.

Palabras clave: Enseñanza de las ciencias sociales, Cultura, Identidad étnica, Diálogo intercultural, Comunicación, Educación.

\section{Abstract}

Think about the way we assume about the ethnic-racial diversity in urban educational institutions. Educators can facilitate the debate about how we understand the cultural differences from the ethnic-racial footprints of theory and its daily manifestations. In that regard, two important tendencies are evident: the first comes from the precept of culture as a homogenous and historical defined category; the second is the one that recognizes the changing character of the culture and the individuals who belong to it. In that way, this document explores the tendencies that lead the debate about teaching the Colombian ethnic-racial differences, through the contents analysis methodology, emphasizing on individual student input, school practices and knowledge that mainly influence the social representations formation. Likewise, we consider the importance of communication-education as the core idea in Intercultural Dialogue processes.

Keywords: Teaching strategies, Cultural context, Ethnic identity, Intercultural dialogue, Communication, Education.

' Magíster en Comunicación-educación, Universidad Distrital Francisco José de Caldas. Docente de Ciencias Sociales. 


\section{INTRODUCCIÓN}

La pregunta por la pertinencia de los saberes científico-sociales, que se enseñan y se construyen desde las instituciones educativas, permite un análisis profundo respecto a la naturaleza misma de los discursos, las acciones y las apropiaciones de los sujetos sobre la realidad social que viven cotidianamente.

Este cuestionamiento, que liga lo práctico a lo epistemológico, se puede dimensionar desde diferentes problemáticas propias del campo de conocimiento. Una de ellas es la comprensión de las diferencias étnico-raciales como esquema explicativo de las tensiones que subyacen los saberes, aproximaciones a los modos de vida y formaciones culturales propias y de otros sujetos sociales. A pesar de los importantes avances teóricos de la enseñanza de las Ciencias Sociales, la sociología de la educación entre otras, las prácticas educativas de contextos escolares parecen apostar a la reproducción de una tradición fosilizada, más que al encuentro de la elasticidad cultural propia de los contextos contemporáneos.

En casos como el colombiano, la enseñanza de las Ciencias Sociales se ha cimentado bajo los valores de un nacionalismo homogéneo y la tendencia a jerarquizar manifestaciones culturales. Ello se hace evidente en estudios y reflexiones provenientes de la Pedagogía (Villa, 2008), la enseñanza de las Ciencias Sociales (Gómez, 2006), la Filosofía (Hoyos, 2010), la Antropología y la Historia (Castro-Gómez y Restrepo, 2010).

Ciertamente, un desafío para la enseñanza de las Ciencias Sociales y los sujetos intervinientes en dicho proceso, es el de conocer y repensar los modos en que se reconoce la cuestión identitaria y la heterogeneidad, en aras de establecer espacios de diálogo intercultural, en oposición a la segregación y al desconocimiento de las particularidades de las culturas presentes en el territorio colombiano.

Para ello, se desarrollará, en principio, una aproximación a los rasgos más característicos de la diferencia étnica y cultural colombiana; en segundo lugar, se expondrán dos enfoques prevalentes en las Ciencias Sociales asociados a la enseñanza de la diferencia étnico-racial; en tercera instancia, se identificará la vinculación de dichos enfoques en experiencias educativas concretas y ,finalmente, se analizará el papel de la comunicación-educación en las transformaciones de prácticas y escenarios excluyentes hacia mecanismos que aboguen por el diálogo intercultural en la educación escolar.

\section{¿CÓMO SE COMPRENDE LA DIFERENCIA CULTURAL COLOMBIANA?}

Colombia posee una característica propia de su ubicación geográfica: disponer de una variedad biológica y geofísica que la hace uno de los lugares con mayor biodiversidad del planeta. Además, esa diversidad se puede trasladar al plano humano. Los pueblos, las sociedades y los sujetos que constituyen el Estado-nación colombiano no son fortuitamente diversos.

La historia de Colombia, así como de la mayoría de países de la región, cuenta con un pasado que explica el porqué de las diferentes expresiones culturales de sus habitantes. Pero es también en el pasado donde se pueden revisitar las formas de invisibilización y exterminio cultural que calan actualmente en el imaginario, los discursos y las formas de representación de las diferencias culturales.

Estas diferencias culturales se manifiestan en los sujetos y en las formas en que se autorreconocen $o$ autoidentifican, de manera que existen ciertas categorías socialmente construidas que develan la pertenencia a una determinada configuración cultural. Estas categorías aparecen en la relación entre sujetos, producto de la jerarquización de la sociedad en la época colonial. En Colombia, históricamente se han apropiado cinco grandes categorías étnico-raciales: blanco, indígena, negro, rom y mestizo.

El blanco es un patrón racial vinculado con la raíz euro-occidental. Es una tendencia social- 
mente privilegiada por considerarse civilizada y culturalmente más avanzada que las otras. Yáñez y Bustamante analizan esta situación de forma acertada cuando indican que "(...) la elite blanco-mestiza, masculina y patriarcal, con una perspectiva eurocéntrica de la historia y la cultura, ha ido configurando su poder (...) y la perpetuación de este, a partir de imaginarios esencialistas que representan la identidad nacional" (20I2, p. 3I).

La categoría indígena tiene igualmente una connotación histórica. El indígena per se (como categoría) no existía antes de la incursión de los europeos a tierras americanas en el siglo XV. Viene de la denominación de indio, también inexistente en la época y que se recrea en los discursos de los peninsulares para denominar al ser que habitaba las tierras de este lado del Atlántico. Desde sus inicios, y hasta ahora, predomina una visión inferiorizada y homogenizada, condenada a la preterización y el exotismo.

En el amplio marco de la sociedad, los diferentes pueblos y comunidades indígenas se han desligado de ese lastre de menosprecio $y$, a través de diferentes movimientos sociales y luchas por el reconocimiento de sus derechos, han logrado importantes conquistas no solo para sus comunidades sino, en general, para el establecimiento y la promoción de una sociedad más justa y equitativa. El desafío está en trasladar esta política de representación a los escenarios educativos.

En cuanto a la categoría de negro, puede tener su génesis en las transacciones comerciales propias del comercio triangular de los siglos XIV y XIV. A las gentes del África subsahariana se les comenzó a denominar negros y bajo ese apelativo, con connotaciones negativas, se le reconoció a los sujetos esclavizados forzosamente, desplazados a territorio americano. Como expresan los estudios de Wade (1996) y Restrepo (1997), hacia los años setenta en Colombia, desde la antropología y la lucha de los movimientos sociales, se empoderaron las categorías de negro y afrocolombiano de forma positiva, aludiendo al carácter de las particularidades ét- nicas y a la reclamación de las demandas fundamentales en términos de derechos frente al Estado. Actualmente, se hace la distinción por aspectos históricos y culturales de la existencia de grupos raizales, habitantes de la región insular colombiana y palenqueros, moradores de uno de los primeros territorios libres de la actual Colombia: el palenque de San Basilio.

En el caso de los rom, al ser una minoría étnica en Colombia, el conocimiento sobre sus particularidades como pueblo no es masivo. El texto de Paternina y Gamboa, Los gitanos: tras la huella de un pueblo nómade (1999), da una idea importante de algunos de sus principales aspectos. La relación de la cultura rom con los integrantes de las sociedades donde se han establecido, ha estado mediada por estereotipos generalmente negativos que impiden un diálogo intercultural.

La identidad, entendida como el elemento que permite diferenciarse de otros grupos humanos, es comprendida desde la perspectiva del pueblo rom en la aceptación, la vigencia y la capacidad de transformación de sus rasgos elementales, a saber: lengua, costumbres, formas de vida y actividades económicas, entre otras; sin embargo, ello no implica que todos los pueblos rom sean homogéneos. Los autores identifican por lo menos tres grandes grupos que se distinguen por cuestiones histórico-geográficas: los cíngaros kalderash, los manuches y los gitanos (Paternina y Gamboa, 1999), subdivididos a su vez en otras denominaciones.

En cuanto a la categoría de mestizo, esta es una de las más deterministas en relación con las anteriormente mencionadas. De acuerdo con Lucía Ortiz, "los indígenas y los negros han sido invisibilizados en una nación que se proyecta idealmente como mestiza y en la cual desaparecen las diferencias de identidad racial" (2007, p. 14); y es que el mestizaje se ha constituido, en ámbitos sociales, como una política de blanqueamiento, que al reconocer nominalmente las diferentes raíces culturales, posiciona lo blanco (hispanoeuropeo) como principal y superior en detrimentos de otras formas culturales. Es 
importante mencionar cómo, además de las categorías referidas, en ámbitos educativos y sociales en general surgen otras formas de autorreconocimiento étnico-racial; entre ellas, una de las más comunes es la categoría de moreno. Esta se asocia frecuentemente a la de mestizo, pero con un tono de piel más oscura. Sandra Soler (20I3), se aproxima a esta situación identificando el papel que juega la "metonimia del color", esto es el color de la piel como referente práctico de las formas de autorreconocimiento étnico-racial.

Más allá de vincular el autorreconocimiento étnico-racial a la cuestión única y exclusivamente del color de piel, los escenarios educativos deben optar por una comprensión de la cultura en sentidos más profundos, que permita la interlocución en espacios de construcción de interculturalidad. De acuerdo con este imperativo, la educación, y en particular las Ciencias Sociales, deben articular esfuerzos desde los sujetos, los discursos y las prácticas escolares hacia la erradicación de la segregación y la diferenciación que subalterniza e inferioriza lo que no está en el plano de lo hegemónico; a saber: el paradigma blanco-mestizo, cristiano de habla hispana.

\section{ENFOQUES DE LA ENSEÑANZA DE LA DIFERENCIA CULTURAL COLOMBIANA}

Las tensiones de carácter étnico-cultural no son ajenas a los contextos educativos escolares; tienen origen cuando "el otro" se objetiviza, se circunscribe en miradas planas de su realidad y se toma ligeramente como inferior o peligroso. Un fenómeno axiomático en este proceso es el de la formación de representaciones sociales, siguiendo la tesis que expresa que "(...) la imagen que nos configuramos del otro, a la distancia, esta mediada por complicados procesos de tipificación: el otro no propiamente se revela, sino que se tipologiza" (Castillejo, 2000, p. 134).

Surge entonces una mirada de lo diferente parcializado, que se refuerza generalmente a través de los discursos y representaciones. "Las representaciones sociales contienen elementos ideológicos, cognitivos y valorativos, y determinan actitudes, estereotipos y otros procesos de los individuos frente a un fenómeno social" (Yáñez y Bustamante, 2012, p. 12). El papel de la educación en la formación de estas representaciones sociales, es un aspecto de suma importancia ligado a la manera en que se asume la identidad. A continuación, se presentan dos enfoques prevalentes en la enseñanza de la diferencia cultural que han incidido sustancialmente en las formas de representación de los sujetos étnicamente diferenciados.

\section{ENFOQUE ESENCIALISTA \\ O DE LA TRADICIÓN FOSILIZADA}

Uno de los retos más apremiantes de los docentes de Ciencias Sociales es compartir la intensidad de las luchas históricas, de las ideas y de las diferentes situaciones, que a lo largo del tiempo han transformado la vida social y las costumbres de los pueblos, tarea poco sencilla que exige ir más allá de las limitaciones de las aulas y del carácter superficial de los textos escolares. Bien sea por las dinámicas internas de las instituciones o por la ausencia de procesos creativos entre los integrantes de la comunidad escolar, lo que sería un escape a pensar al sujeto como constructor de la historia, se vuelve una tediosa tarea repetitiva y carente de sentido.

Para el caso de la aproximación a las identidades y a las culturas colombianas, se acude a lo que Salas Astrain (2010) ha de denominar "tradición fosilizada", y es el cierre de posibilidades a voces distintas a las hegemónicas. Ciertamente, estas versiones de la historia, y de las problemáticas específicas de las Ciencias Sociales, imposibilitan un diálogo y una construcción diversa de fenómenos, para este caso, de carácter cultural. Ello ya supone de entrada la naturalización de las diferencias étnico-raciales, en la medida en que, si bien es cierto que se identifican algunos rasgos de los grupos humanos, se desconoce el carácter de construcción social de conceptos como raza, a los que generalmente se les otorga un origen biológico, tema que han develado con 
cierta notoriedad los grupos de estudios poscoloniales y decoloniales (Verrangia, 2014).

Ya en el terreno propiamente de la identidad, se puede afirmar que las diferentes sociedades y grupos humanos se han caracterizado por constituir formas de pertenencia y autorreconocimiento que les diferencian de otros (Geertz, 1996). Sin embargo, estos sentimientos de pertenencia no siempre son equiparables a las formaciones culturales, a las que se les suele asignar un carácter más universalizante, en la medida en que la identidad se asocia a lo contingente; es, en palabras de Martín-Barbero, “(...) una dimensión del lazo social, cada vez más fracturada e individualista" (2004, p. 35).

Para diferenciar la identidad de lo cultural, Grimson define que: "Lo identitario refiere a los sentimientos de pertenencia a un colectivo y a los agrupamientos fundados en intereses compartidos" (201I, p. 138). Esta acepción permite entender cómo la cuestión de adhesión a un grupo es inherente a los seres humanos, característica que comparte con la cultura, aunque, en el plano de lo étnico-racial, lo identitario está atravesado tanto por la deshistorización, la ausencia de sentido y de pertenencias, como por la parcialización o la ideologización de una realidad histórica.

Esta primera tendencia, a la que se ha denominado tradición fosilizada, también puede ser interpretada como una visión esencialista de la identidad, que desconoce los cambios presentes en los grupos y sujetos pertenecientes a una determinada sociedad y que, por el contrario, aboga por una añoranza y exaltación de mitos fundacionales, que al ser fuente de análisis de las realidades presentes de las culturas, suele ser un modelo obsoleto en sí mismo.

La perspectiva esencialista comparte un modelo de identidad que solo comprende las relaciones endógenas de cada cultura; ejemplo de ello son las tesis del antropólogo mexicano Guillermo Bonfil Batalla (1995), en las que hace un llamado a definir la identidad a partir de la herencia soslayada de los pueblos indígenas. EI intelectual mexicano señala que se debe volver a aquello arrebatado por la colonización y deja entrever, en sentido negativo, la llegada de gentes europeas al continente americano. Lo anterior se explica de la siguiente manera:

La cultura europea no habría sido entre nosotros más que una yuxtaposición colonial y neocolonial, una cultura imaginaria o una apariencia de modernidad. Deberíamos volver a nuestras raíces ocultas bajo este mundo impuesto por los dominadores extranjeros, encontrando allí nuestra única y verdadera identidad (Vergara et al., 2010, p. 66).

Bajo esta misma perspectiva, se puede hallar lo que Rascón denomina identidades particularistas, que emergen en la medida en que “(...) se reduce la identidad a uno solo de sus elementos, pues este únicamente es compartido por un grupo pequeño de personas si lo comparamos con aquel que resulta al concebir nuestra identidad en toda su amplitud" (2006, p. 53).

Otro académico que hace referencia a este enfoque es Oscar Del Álamo, que, en su tesis doctoral El regreso de las identidades perdidas (2007, p. 7I), denomina "enfoque primordialista" a aquellos estudios en los que la identidad étnica se configura como algo natural o primario (p. 7I); de igual manera, se caracteriza una versión instrumental de la identidad cuando se redime solo para la obtención de ciertos beneficios en el campo político: "La identidad no es la que cobra relevancia sino el interés común del grupo o los intereses de una élite que manipula la identidad grupal para propósitos políticos" (Del Álamo, 2007, p. 72).

Existe cierto consenso respecto a lo problemático e inconveniente de tomar la identidad de forma univoca, pues uno de sus más considerables efectos es la ausencia de memoria, en la medida en que las diferentes comunidades $y$ sociedades a lo largo de la historia se han relacionado de forma pacífica o violenta $y$, en ese devenir, la identidad de los sujetos culturales se transforma, apropia y replantea lo que se puede denominar la esencia de su identidad. No es 
adecuado, por ello, señalar que la identidad permanece siempre fija y estática (Restrepo, 2004), modo en el cual se establecen procesos de relacionamiento y acercamiento a diferentes pueblos, especialmente indígenas, afro y rom, en el caso colombiano.

Otro discurso fuertemente arraigado es el de la igualdad. Si bien es cierto que el avance en el reconocimiento de derechos de las comunidades, consideradas como minorías étnicas, ha sido importante en la historia de Colombia durante las últimas décadas, no es menos cierto que los discursos sobre la igualdad tienden a relativizar y dejar de lado los matices identitarios sobre los que se cierne la particularidad de los sujetos.

\section{DEL ENFOQUE RELATIVISTA}

\section{A LA DINÁMICA DE LA DIFERENCIA CULTURAL}

La pretensión de universalidad propia de las tendencias liberales en las Ciencias Sociales, que tomó importante fuerza hacia el siglo XIX (Wallerstein, 1995), buscó dirimir, en cierto sentido, los conflictos y profundas divisiones sociales, apelando a la idea de igualdad en términos jurídicos. La cuestión de la igualdad podría tomarse como un aspecto esencial en la abolición de empresas oprobiosas como la esclavitud y el trato nada benigno hacia las comunidades originarias de América. Sin embargo, varios autores coinciden en que la universalidad y la igualdad, en términos identitarios, acaban por desconocer los rasgos particulares y el carácter de autenticidad de los pueblos (Nivón, 2013).

Este enfoque en sí mismo es paradójico: sus discursos son una invitación a la aceptación de la diferencia aunque, por otra parte, su llamado a la igualdad desfigura las particularidades de los procesos identitarios de los sujetos. Dicha perspectiva, propia de los discursos que legitimaban la formación de los estados-nacionales latinoamericanos durante el siglo XIX, se puede relacionar a su vez con el paradigma del relativismo cultural. Cameron McCarthy, en un análisis de este paradigma en la educación, advierte que su incidencia "(...) se traduce en orientaciones curriculares para los estudios étnicos en términos de un discurso de reciprocidad y consenso: somos diferentes pero todos somos iguales" (McCarthy, 1994, p. 56).

Este discurso relativista ha dado lugar a que, por ejemplo, categorías de identificación étnico-racial, como el mestizaje, se enarbolen como plurales, pero tengan un trasfondo hegemónico a la vez. De lo anterior, surge el desafío de comprender que si la identidad nace de un encuentro de diferencias entre un yo y los otros, debe llevar a reconocer, según Bari, que:

“(...) No es el aislamiento lo que crea la conciencia de pertenencia, sino que es la historicidad de las relaciones de los grupos minoritarios con la estructura de la sociedad global, de donde surge lo distintivo de lo étnico" (Bari, 2002, p. 157).

La identidad étnica, al formar parte del mundo social, no puede desligarse de las históricas relaciones que se han establecido entre culturas y que son los escenarios donde se propician los cambios, las asimetrías y los procesos de complejización de la cuestión étnica.

Posturas como la de María Cristina Bari, generan un punto de partida para considerar el potencial de la dinámica de las identidades. Adriana Márquez, en su tesis, Clima social y autoeficacia percibida en estudiantes inmigrantes, considera que "(...) evidentemente como las culturas no son estáticas, las identificaciones son transferibles, pueden evolucionar y aculturarse, sin perder lo aprendido sino sumando nuevos elementos" (2004, p. 72). Conviene, entonces, profundizar en el enfoque de corte fenomenológico, si se quiere, en cuanto rescata la centralidad del sujeto en los procesos de formación identitaria.

Pasar de una visión plana de las diferencias al debate por las tensiones y los quiebres en la construcción de identidad y alteridad en contextos educativos, es el paso a la reconstitución de 
saberes y haceres. Tener presente una perspectiva dinámica de la identidad, supone desmantelar las formas en que docentes y estudiantes se aproximan al estudio de las diferencias étnico-raciales en la escuela. Es, en este enfoque, en el que se pueden ubicar posturas que analizan la identidad desde la interculturalidad crítica. Catherine Walsh (2007), por ejemplo, aboga por una interculturalidad decolonizada, la diversificación de los saberes y los conocimientos en oposición a la predominancia académica de corte eurocéntrico.

Claudia Vargas, en la tesis Estrategias didácticas para el desarrollo de la identidad cultural Mochica en educación primaria en una Institución Educativa de San José de Moro, La Libertad, comprende la identidad como: “(...) El sentimiento positivo y de pertenencia que tienen las personas o grupos de personas hacia un conjunto de características, experiencias y herencias sujetas a un proceso histórico de cambio y transformación" (2012, p. 17). Esta mirada dinámica, en contraposición al estatismo del enfoque esencialista, también se halla en estudios como el de Belkis Castro, en el que coincide en caracterizar la identidad nacional colombiana como: “(...) Un esquema figurativo que muestra elementos comunes en los diferentes escenarios de la colombianidad" (2012, p. 102). Dicho planteamiento evidencia que no existe un patrón común en la configuración de la identidad.

Lo anterior, visto en términos de construcción de ciudadanía, adquiere especial importancia cuando se habla de una formación de ciudadanía incluyente y democrática, por cuanto estas identidades múltiples "(...) no solo buscan reconocimiento y legitimación, sino que ellas mismas se asumen como poseedoras de unas concepciones de mundo propias, de unas normativas que las constituyen y reconfiguran" (Gómez, 2006, p. 189).

Oscar del Álamo reconfigura desde esta perspectiva la noción de identidad étnica asumiéndola como "(...) un proceso socialmente construido y estructurado" (2007, p. 77). Algunos autores, antropólogos fundamentalmente, asumen una postura crítica de las identidades étnicas; es el caso de Michel Agier, quien señala en Identidad cultural, identidad ritual, que la identidad “(...) no es un hecho dado sino más bien algo que puede llegar a existir en algunos momentos, en ciertas ocasiones" (2002, p. 296). Si bien este juicio se enmarca dentro del enfoque dinámico, tiende a relativizar la identidad étnica y cultural en la medida en que se asume que no hay una identidad fija, sino que en buena parte depende de lugares y situaciones que redimensionan las pertenencias de los sujetos.

A modo de balance, los autores expresan la necesidad de superar la acriticidad y el dogmatismo presentes en algunas perspectivas $y$ es por ello que defienden el análisis de la identidad desde una perspectiva de las interacciones sociales y culturales, alejada de una visión estática de la realidad social. La identidad, más allá de ser un objeto de estudio predominante de la sociología y la antropología, debe considerarse en escenarios frecuentemente relacionados con la reproducción de ideologías hegemónicas como lo son los escenarios escolares.

Asimismo, el hecho de reconocer lo polivalente de la categoría, no significa que sea acertado depreciar los esfuerzos por construir bases sólidas, alejadas de las prenociones y juicios que, por el contexto histórico, se hacen muy perceptibles en los diferentes desarrollos teóricos estudiados. En concordancia con los intereses de la investigación, se apropiará esta perspectiva de la identidad: como una categoría dinámica en permanente diálogo con el pasado pero también con el presente.

Su carácter dinámico o acrítico se construye, en considerable medida, en las instituciones educativas que, como ya se mencionó, pueden ser el lugar de la reproducción o de la emancipación (Dietz, 2012). Es en este punto, donde adquieren relevancia las contribuciones del campo de la comunicación-educación ${ }^{2}$, la cuestión de

${ }^{2}$ El profesor Juan Carlos Amador define la categoría de campo a partir de los postulados de Bourdieu como “(...) espacios sociales y simbólicos que son asimilados por los agentes a través de procesos crecientes de socialización” (Amador, 2014, p. 60). 
la identidad y la cultura forma parte de sus enclaves epistemológicos y permite ver los fenómenos más allá de las fronteras disciplinarias tan arraigadas dentro de las Ciencias Sociales.

La comunicación-educación, como campo de conocimiento, se piensa en los horizontes del reconocimiento del mundo cultural, tanto en el reconocimiento de sí, como de la apropiación de la acción dialógica en el conocimiento de los otros (Huergo, 2013).

Pensar entonces los problemas sociales desde el referente de lo comunicativo/educativo, implica deslindar las fronteras y las certezas hasta ahora infructuosamente imperantes en las dinámicas propias de las Ciencias Sociales escolares, para dar lugar a actos creativos, tanto teóricos como metodológicos, en el abordaje de fenómenos sociales revestidos de perenne complejidad, como la identidad y la cultura. En palabras del profesor Raúl Fuentes Navarro:

Desde esta perspectiva plenamente sociocultural, rearticular los procesos subjetivos e intersubjetivos de significación, a través de los esquemas perceptuales e interpretativos que en cada sector cultural median las relaciones posibles con las estructuras y los sistemas objetivos de procesamiento y difusión de la información, es una clave que, además de restituir la complejidad de los procesos socioculturales en los modelos de comunicación, puede servir para enfatizar la agencia o la acción transformadora implícita en las prácticas de comunicación, es decir, en la interacción material y simbólica entre sujetos concretamente situados, que supone la recurrencia por parte de ellos tanto a sistemas informacionales como a sistemas de significación, cuya competente mediación determina la producción y la reproducción del sentido: el de las prácticas socioculturales de referencia y el de la comunicación misma (Fuentes, 1999, p. 60).

De acuerdo con lo expresado, otorgar un nuevo sentido a la comprensión de la identidad étnica y de las construcciones identitarias propias y de los demás, significa abrir espacios para transformar las relaciones mismas entre los integrantes de una comunidad escolar; y no solo ello, sino permitir a la vez trascender las fronteras de la escuela, articulando los procesos macrosociales con los procesos escolares. En los términos de Jorge Huergo y María Belén Fernández:

La comunicación en educación trabaja postulando a los sujetos como autónomos (como punto de partida) para que, en la conquista y desarrollo de su autonomía, instituyan una sociedad autónoma con individuos autónomos, que rebasen la expectativa de la funcionalidad, organización racional, eficiencia, claridad y distinción, y que construyan la autonomía: imposibilidad lógica a la que integra y radical posibilidad creativa (Huergo y Fernández, 1999, p. 134).

\section{SESGO Y DISGREGACIÓN}

\section{EN LA ENSEÑANZA DE LAS DIFERENCIAS ÉTNICO-RACIALES COLOMBIANAS}

Uno de los peligros de naturalizar los conflictos sociales consiste en perder de vista su carácter histórico y las dinámicas culturales de poder que subyacen los diferentes encuentros de grupos poblacionales. Ello sucede cuando se indaga respecto a situaciones como la aculturación colonial en la que, de entrada, ya se inferioriza al sujeto indígena o se convierte en motivo de burla y exotización en la medida en que se considera diferente.

El sesgo viene dado por varias circunstancias. En primer lugar, por un fenómeno al que Jan Gustafsson (2004) ha denominado preterización, y es el hecho fáctico de deshistorizar a una comunidad en particular, producto de las hondas raíces que el colonialismo cierne sobre los saberes y conocimientos que se reproducen desde las Ciencias Sociales escolares; a la par, se pone de manifiesto la desarticulación entre los avances teóricos y discusiones contemporáneas sobre el fenómeno y los procesos de enseñanza-aprendizaje en los niños y jóvenes de las instituciones educativas. 
Este aspecto suele ser más frecuente en contextos urbanos donde las diferencias culturales se tienden a relativizar cuando no a anular. En el caso de instituciones en contextos rurales se puede apreciar un significativo avance que, aunque no se hace extensivo a todos los casos, sí se evidencia en procesos ejemplarizantes de la posibilidad de cambio desde la construcción de otras formas de pensar la enseñanza y la aproximación a los conocimientos científico-sociales. Un caso de importante trayectoria es el de la Normal Superior de los Montes de María, en el municipio de San Juan Nepomuceno, departamento de Bolívar, en el que se ha hecho una importante labor de reconstrucción de la memoria histórica de los habitantes del municipio y revalorización de sus posibilidades como sujetos históricos. Otro caso, desde la experiencia de pueblos afrocolombianos en el norte del Cauca, es el de la Asociación Casita de Niños y del Colegio Valentín Carabalí, en el que la tradición, lejos de ser un elemento anquilosado en un pasado inerte, permite la comprensión del presente y las posibilidades en el futuro desde las particularidades de sus trayectorias étnico-raciales.

Las identidades étnico-raciales colombianas en las Ciencias Sociales aparecen como un fenómeno disgregado, del cual se tiene razón por hechos históricos aislados en los que se anula el conflicto. No obstante, efemérides como la del día de la independencia, 20 de julio y la del 12 de octubre, desafortunadamente señalado aún como el "día de la raza", suelen exaltar una historia basada en los cánones de la historia oficial, olvidando los procesos de lucha y paradójica irracionalidad del proyecto civilizatorio ilustrado del siglo XIX (Rueda, 200I).

La consecuencia de ello es pensar que la realidad de los pueblos indígenas es un asunto del pasado, de modo tal que se desconocen las particularidades que hay en cada uno de los pueblos originarios que habitan en el actual territorio colombiano, además de las copiosas representaciones gráficas de cuerpos semidesnudos y flechas, o cuerpos con rasgos exageradamente marcados en la representaciones de personas con raíces afrocolombianas. Desde una pers- pectiva axiológica, se ha optado por incentivar la tolerancia, más que el conocimiento y la necesidad de repensar los esquemas de diálogo y relacionamiento con los otros, la subalternización de la historia oficial y la revictimización por parte de la enseñanza de las Ciencias Sociales y los saberes que allí circulan a causa del desconocimiento.

Ante esta realidad, varios autores han dispuesto una serie de estrategias; entre ellas, una pedagogía intercultural para contextos urbanos (Pinzón, 2010), la de-colonización de las formas de conocimiento escolar (Rojas, 2010), y la transformación de la construcción discursiva de las identidades en los libros de texto (Yáñez y Bustamante, 2012). Estas voces concuerdan en una necesidad de cambio, aunque ello solo es posible con voluntad de parte de los integrantes de la comunidad educativa $y$, fundamentalmente, de los procesos que se inicien desde el campo de conocimiento de las Ciencias Sociales, en cuyo acontecer recae la responsabilidad de diálogo, negociación y prácticas más cercanas a la realidad social.

\section{CONTRIBUCIONES DEL CAMPO}

\section{COMUNICACIÓN-EDUCACIÓN AL}

\section{PLURALISMO HISTÓRICO Y CULTURAL}

Siguiendo la premisa de Jesús MartínBarbero (2003), sobre el carácter de transversalidad de los saberes, se considera que la comunicación-educación es el campo de conocimiento estratégico para delinear la cartografía de los intersticios de la relación identidad-cultura-educación. Los aportes del campo comunicación-educación, transversalizan el problema de investigación y de su interior devienen debates, contextos y posturas críticas de carácter político que constituyen, como primordial, la acción y apuesta por el cambio social desde la creatividad, la transformación cultural y no solamente desde lo instrumental de la cultura.

Ejemplos de ello son las estrategias de enseñanza que brinda el campo y que se pueden adoptar en la elaboración de un nuevo marco 
de comprensión de los fenómenos sociales en la educación. Una de ellas es la reflexión sobre las salidas a la pretensión de homogenización cultural. El antropólogo y teórico del campo de la comunicación-educación Néstor García Canclini (2005), en su análisis de las culturas latinoamericanas, aporta un significativo paso hacia el conocimiento y reconocimiento de la pluralidad y las divergencias socioculturales de los pueblos de la región. Frente a la histórica pretensión de homogenización cultural y el emplazamiento del proyecto moderno en la mayor parte de las instancias sociales de siglos pasados, la hibridez es una ruptura que da lugar a formas estéticas, académicas y políticas fuera de los cánones de la monoculturalidad hispano-occidental del centro y sur de las Américas.

No dejan de ser, por lo tanto, formaciones culturales; lo son en el sentido de disputas y encuentros de lo culto y lo popular, lo tradicional y lo moderno, lo hegemónico y lo subalterno. Estos diálogos interculturales que se configuran en hibridez cultural no disuelven el conflicto de fondo, pero son modelos tangibles de desmantelamiento de un proyecto único y avasallador de sociedad. Luego, ante la pregunta de cómo ello permea, se extiende, crea o recrea en instituciones educativas formales, es un desafío en la consolidación de estrategias de enseñanza enfocadas a ampliar el marco interpretativo de las culturas y de la construcción de identidades étnico-raciales.

Otro aspecto de primer orden del que se ocupa el campo de estudios de la comunicación-educación es la superación de las temporalidades tradicionales.

El tiempo lineal no explica en su totalidad la causalidad, el desarrollo y el devenir de los procesos culturales. Jesús Martín-Barbero, consciente de ello, en sus múltiples publicaciones señala, ya no el tiempo, sino las heterogeneidades temporales de sociedades y sujetos, despojando de la unicidad de criterio a las formaciones culturales que puedan existir en una $\circ$ varias espacialidades.
Al hacer referencia a las heterogeneidades temporales, se procura dejar en evidencia las transformaciones culturales de la época contemporánea y que el cambio escolar no esté desligado del cambio social.

Lo anterior cuestiona la enseñanza de la Historia y de las Ciencias Sociales desde temporalidades dominantes, pero al tiempo encuentra quiebres que son posibles a través de la voluntad docente y del asumir una postura ética-política frente a la vocación desempeñada y al campo de conocimiento en el que se mueve.

\section{CONCLUSIONES}

A través de esta reflexión se ha podido establecer la existencia de los enfoques prevalentes al momento de pensar en la enseñanza de la diferencia étnico-racial colombiana. En líneas generales, la reproducción de ciertos saberes escolares esencialistas de las Ciencias Sociales, han dejado como huella un profundo desconocimiento de la realidad histórica que conlleva a un conocimiento sesgado y prejuicioso de los grupos étnicos-raciales contemporáneos, constituyentes de uno de los elementos básicos del Estado colombiano: la estructura de su población.

Asimismo, se reconoce que, además de las contribuciones de diferentes campos del conocimiento de las Ciencias Sociales, la comunicación-educación, permite la rearticulación de la experiencia histórica con los movimientos y nuevas formas de relacionamiento de los sujetos en las sociedades del siglo XXI. A todo ello se suma, en última instancia, que no son suficientes las vastas elaboraciones teóricas, si no existe, de parte de los integrantes de la comunidad educativa (en concurso con las disposiciones de instituciones estatales y organizaciones sociales), una verdadera voluntad de transformación, no solo del saber sino de las prácticas comunicativas dentro y fuera de las instituciones educativas. 
En un país donde la diferencia se ha de dimensionar como una potencialidad, es necesario el ejercicio de establecer diálogos interculturales, como nueva semántica cultural en lo educativo y social. En este sentido, la comunicación ha de ser su fundamento por cuanto posibilita nuevas formas dialógicas en los sujetos. Esta es una apuesta por la aproximación a diferentes modos de vida, no desde la nostalgia de lo atávico, sino desde las formaciones culturales contemporáneas, subjetividades en permanente realización, tal como lo indica Ricardo Salas Astrain “(...) en medio de un mundo económico donde los mercados y las culturas interactúan y donde los mundos de vida se entrecruzan e interpelan" (2006, p. 22).

Ello no será posible si no se replantea el modo en que los docentes exponen los sabe- res sociales escolares, pues aunque los avances desde los campos de conocimiento científicos son importantes, esto no se evidencia plenamente en las instituciones educativas escolares en las que se sigue primando lo episódico y unas tradiciones culturales carentes de sentido. Se aprende una historia por obligación y no para la transformación. Finalmente, la comunicación-educación, como campo de conocimiento, dispone de reflexiones que pueden ir al encuentro de dinámicas transformadoras pertinentes, en un despliegue de rearticulaciones de la experiencia histórica colombiana con la experiencia social cotidiana, en la que los tiempos, los destiempos, las hibridaciones y los mestizajes formen parte de las subjetividades constituyentes de los estudiantes y de quienes comparten su entorno \&

\section{Referencias bibliográficas}

Agier, M. (2002). Identidad cultural, identidad ritual: una comparación entre Brasil y Colombia (Mosquera, C. Trad.). En: Mosquera, Pardo y Hoffmann (Eds.), Afrodescendientes en las Américas: trayectorias sociales e identitarias. Bogotá: Instituto Colombiano de Antropología e Historia -ICANH, Universidad Nacional de Colombia. (Obra original: Identité culturelle, identité rituelle. Une comparaison Brésil/Colombie, 200I).

Amador, J. (2014). Infancias, comunicación y educación: análisis de sus mutaciones. Bogotá: Fondo de publicaciones Universidad Distrital Francisco José de Caldas. 
Bari, M. (2002). La cuestión étnica: aproximación a los conceptos de grupo étnico, identidad étnica, etnicidad y relaciones interétnicas. Cuadernos de Antropología Social, 16, pp. 149-163. Versión en línea, recuperada el 27 de agosto de 2013, de: http://www.scielo.org.ar/ scielo.php?pid=S1850-275X2002000200002\&script $=$ sci_arttext

Bonfil, G. (1995). Obras escogidas. México, D. F.: Instituto Nacional Indigenista.

Castillejo, A. (2000). Anatomía de la intimidad. En: Restrepo, E. y Uribe, V. (Comp.) Antropologías transeúntes. Bogotá, D.C.: Instituto Colombiano de Antropología e Historia -ICANH, Universidad Nacional de Colombia.

Castro, B. (2012). Las representaciones sociales de la identidad nacional de los colombianos (Tesis de Maestría en Investigación Social Interdisciplinaria). Universidad Distrital Francisco José de Caldas, Bogotá, D.C., Colombia.

Castro-Gómez, S. y Restrepo, E. (Ed.) (2008). Genealogías de la colombianidad. Bogotá: Instituto Pensar, Pontificia Universidad Javeriana. Del Álamo, O. (2007). El regreso de las identidades perdidas: movimientos indígenas en países centro-andinos (Tesis doctoral). Recuperado el 2 de junio de 20I4, de: http://www.tdx.cat/bitstream/handle/I0803/724l/toap.pdf?sequence =I .

Dietz, G. (2012). Multiculturalismo, interculturalidad y diversidad en educación. México, D.F., Fondo de Cultura Económica.

Fuentes, R. (1999). La investigación en comunicación en América Latina: condiciones y perspectivas para el siglo XXI. Diálogos de la comunicación, 56, 52-67.

García C. (2005). Culturas híbridas: estrategias para entrar y salir de la modernidad. México, D.F:: Grijalbo.

Geertz, C. (1996). Los usos de la diversidad. Barcelona: Paidós.

Gómez, J. (2006). La formación de sujetos sociales y el aprendizaje ciudadano. En: Estructura, tiempo y sujeto. Nuevos recursos para la discusión interdisciplinaria. Bogotá, D.C.: Editorial Universidad Distrital Francisco José de Caldas.

Grimson, A. (20I I). Los límites de la cultura: crítica de las teorías de la identidad. Buenos Aires: Siglo Veintiuno Editores.

Gustafsson, J. (2004). El cronotropo cultural, el estereotipo y la frontera del tiempo: la preterización como estrategia de representación del otro. Cultura, lenguaje y representación I, I37-147.

Hoyos, G. (2010). Enseñanza de las Ciencias Sociales: su papel en la consolidación de una sociedad incluyente, participativa y democrática. En Camilo Gonzáles Posso (Presidencia), Ciclo de conferencias para docentes del Distrito "Memoria con sentido de futuro", Centro de Memoria, Paz y Reconciliación, Instituto para la Investigación Educativa y el Desarrollo Pedagógico -IDEP. Bogotá, D.C., Colombia.

Huergo, J. (diciembre, 2013). Mapas y Viajes por el campo comunicación-educación. Tram(p)as de la comunicación y la cultura, 75, 19-30.

Huergo, J., y Fernández, M. (1999). Cultura escolar, cultura mediática/ Intersecciones. Bogotá, D.C.: Colegio Académico de Comunicación y Educación, Universidad Pedagógica Nacional.

Márquez, A. (2005). Clima social y autoeficacia percibida en estudiantes inmigrantes: una propuesta intercultural (Tesis doctoral). Recuperado el 10 de mayo de 20I4, de: http://eprints.ucm.es/tesis/edu/ucm-t28025.pdf

Martín-Barbero, J. (mayo-agosto, 2003). Saberes hoy: diseminaciones, competencias y transversalidades. Revista lberoamericana de Educación 32. Recuperado el 4 de abril de 2014, de: http://es.scribd.com/doc/6334183/Saberes-hoy-transversalidades-competencias-y-diseminaciones

Martín-Barbero, J. (2004). Crisis identitarias y transformaciones de subjetividades. En Laverde M. C., Daza, G. y Zuleta, M. (Ed.), Debates en torno al sujeto: Perspectivas contemporáneas. Bogotá, D.C.: Siglo del Hombre Editores.

McCarthy, C. (1994). Racismo y curriculum: la desigualdad social y las teorías y políticas de las diferencias en la investigación contemporánea sobre la enseñanza. Madrid: Fundación Paideia, Ediciones Morata.

Nivón, E. (20/3). Las políticas culturales en América Latina en el contexto de la diversidad. En: Hegemonía cultural y políticas de la diferencia. Versión en línea, Recuperada el 2 de abril de 2014, de: http://biblioteca.clacso.edu.ar/gsdl/collect/clacso/index/assoc/D863I.dir/ eduardo_bolan.pdf

Ortiz, Lucia. (2007). Chambacú: la historia la escribes tú: ensayos sobre cultura afrocolombiana. Madrid: Iberoamericana Vervuert Verlag. Paternina, H. y Gamboa, J. (1999). Los gitanos: tras la huella de un pueblo nómade. Revista Nómadas, (10). I56-I70. Recuperado el I5 de septiembre de 2013, de: http://www.ucentral.edu.co/images/stories/iesco/revista_nomadas/I0/nomadas_I0_I2_los_gitanos.pdf Pinzón, M. (septiembre-octubre, 2010). Anotaciones para una pedagogía intercultural en contextos urbanos. Revista internacional Magisterio, 46, 28-3I.

Rascón, M. (2006) La construcción de identidad cultural desde una perspectiva de género: el caso de las mujeres marroquíes (Tesis doctoral). Universidad de Málaga, España. Recuperado el 20 de abril de 2014, de: http://www.biblioteca.uma.es/bbldoc/tesisuma/16795246.pdf 
Restrepo, E. (1997). Afrocolombianos, antropología y proyecto de modernidad en Colombia. En: Uribe, M. V. y Restrepo, E. (Eds.) Antropología en la modernidad: identidades, etnicidades y movimientos sociales en Colombia. Bogotá, D.C., Instituto Colombiano de Antropología-ICAN.

Restrepo, E. (2004). Teorías contemporáneas de la etnicidad Stuart Hall y Michel Foucault. Popayán: Editorial Universidad del Cauca. Rojas, A. (2010). El sistema mundo moderno como contexto de emergencia de la diáspora. En: Colombia afrodescendiente: lineamientos curriculares de la cátedra de estudios afrocolombianos 2010, Bogotá, D.C.: Ministerio de Educación Nacional -MEN.

Rueda, J. (200I). Las ciencias sociales colombianas y el imaginario de nación: Henao y Arrubla o la perpetuación de la visión académica. En: Rueda, J., y Serna, A. (Comp.) Investigación y cultura política. Bogotá: Centro de Investigaciones y Desarrollo Científico, Universidad Distrital Francisco José de Caldas.

Salas, R. (2010). Ética intercultural: ensayos de una ética discursiva para contextos culturales conflictivos. (Re) Lecturas del pensamiento latinoamericano. Quito: Ediciones Abya-Yala.

Soler, S. (2013). Entre negro oscuro y moreno claro: discursos e identidades étnicas en niños y niñas afrodescendientes en contexto escolar en Bogotá. Educar em Revista, 47, III-I43. Recuperado el 5 de mayo de 2014, de: http://www.scielo.br/scielo.php?pi$\mathrm{d}=$ S0 I04-406020 I3000 I00009\&script $=$ sci_arttext

Vargas, C. (2012). Estrategias didácticas para el desarrollo de la identidad cultural Mochica en educación primaria en una Institución Educativa de San José de Moro - La Libertad (Tesis de maestría). Recuperado el 10 de mayo de 20I4, de: http://tesis.pucp.edu.pe/repositorio/ bitstream/handle/I23456789/4574/NARGAS_ORTIZ_DE_ZEVALLOS_CLAUDIA_ESTRATEGIA_MOCHICA.pdf?sequence= I

Vergara, J. I., Vergara, J. y Gundermann, H. (2010). Elementos para una teoría crítica de las identidades culturales en América Latina. Utopía y Praxis Latinoamericana. I5(5I), 57-79. Recuperado el I de septiembre de: http://www.scielo.org.ve/pdf/upl/vI5n5l/art05.pdf

Verrangia, D. (20l4). Multiculturalismo, relaciones etnoraciales y enseñanza de las ciencias: algunos desafíos. En: Mosquera, C. (Comp.), Miradas contemporáneas en educación: algunos puntos clave para el debate. Bogotá, D.C.: Fondo de Publicaciones, Universidad Distrital Francisco José de Caldas.

Villa, W. (2008). La cultura en el marco de la educación: acciones posibles para una construcción de lo otro. Educación y cultura 79, 62-68.

Wade, P. (1996). Identidad y etnicidad. En: Escobar, A. y Pedrosa, A. (comp.) Pacífico idesarrollo o diversidad: Estado, capital y movimientos sociales en el Pacífico colombiano. Bogotá, D.C: Ecofondo-CEREC.

Wallerstein, I. (1995). Abrir las ciencias sociales. México, D.F:: Siglo veintiuno Editores.

Walsh, C. (2007). ¿Son posibles unas ciencias sociales/culturales otras? Nómadas 26, 102-I I3. Versión en línea, recuperada el 6 de febrero de 2014, de: http://www.ram-wan.net/restrepo/decolonial//6-walsh-son\%20posibles\%20unas\%20ciencias\%20sociales\%20culturales\%20otras.pdf

Yáñez, C. y Bustamante, U. (20I2). Identidades y alteridades en Colombia: su construcción discursiva a través de la historia. Manizales: Universidad Nacional de Colombia. 